\title{
Objetivos e desafios da política de compras públicas sustentáveis no Brasil: a opinião dos especialistas
}

\author{
Hugo Leonnardo Gomides do Couto \\ Fundação Instituto Brasileiro de Geografia e Estatística (IBGE) / Unidade Estadual em Goiás \\ Goiânia / GO - Brasil \\ Francis Lee Ribeiro \\ Universidade Federal de Goiás (UFG) / Programa de Pós-Graduação em Ciências Ambientais \\ Goiânia / GO - Brasil
}

\begin{abstract}
O presente artigo investiga como os especialistas compreendem, refutam ou validam os objetivos e estabelecem os desafios prioritários à implementação da política pública de compras sustentáveis na esfera federal. Para tanto, foi utilizado um Delphi de políticas associado à análise de conteúdo, para explorar as variadas opiniões associadas à política pública e construir categorias para sua análise, sendo útil como ferramenta de apoio à decisão do governo brasileiro. Verifica-se que os especialistas compreendem essa política como de cunho ambiental em sentido estrito e ratificam a necessidade de integração das diferentes políticas públicas que atuam em prol da sustentabilidade. Adicionalmente, identifica-se que as barreiras envolvendo aspectos institucionais (cultura organizacional resistente a mudanças, ausência de capacitação etc.) e questões ligadas ao aprimoramento dos mecanismos de informação aos gestores públicos (elaboração de guias práticos, esquemas de rotulagem e certificação ambiental etc.) constituem os principais desafios à adequada efetivação da política pública.
\end{abstract}

Palavras-chave: compras públicas sustentáveis; Delphi de políticas; política pública; análise de conteúdo.

Objetivos y desafíos de la política de compra sostenible en Brasil: la opinión de expertos Este artículo investiga cómo los expertos entienden, refutan o validan los objetivos y establecen los desafíos prioritarios para la aplicación de la política pública de compras sostenibles a nivel federal. Por lo tanto, se utilizó un Delphi de políticas asociados con el análisis de contenido, para explorar las diversas opiniones relacionadas con la política pública y crear categorías para su análisis, siendo útil como herramienta de apoyo a las decisiones del gobierno brasileño. Parece que los expertos entienden la

DOI: http://dx.doi.org/10.1590/0034-7612146561 
política como siendo estrictamente ambiental y confirman la necesidad de integración de las diferentes políticas públicas que trabajan por la sostenibilidad. Además, identifica que las barreras asociadas con los aspectos institucionales (cultura organizacional resistentes al cambio, la falta de formación de los funcionarios públicos etc.) y las cuestiones relacionadas con la mejora de los mecanismos de información a los gestores (desarrollo de guías prácticas, sistemas de etiquetado y certificación ambiental etc.) son los principales desafíos para la correcta ejecución de la política pública.

Palabras clave: compras públicas sostenibles; método Delphi; políticas públicas; análisis de contenido.

Objectives and challenges of the sustainable public procurement policy in Brazil: expert opinions This article investigates how experts understand, refute or validate the objectives and establish the overriding challenges for the implementation of the sustainable procurement public policy at federal level. For that, a policy Delphi associated with content analysis was used in order to examine the varied opinions related to that public policy, and to build categories for their analysis, which is useful as a support tool for Brazilian government's decision-making. It was found experts understand that policy as having a strict environmental nature, and they confirm the need for integration of different public policies that work for sustainability. In addition, it may be perceived that barriers involving institutional aspects (an organizational culture resistant to change, lack of public servants' training, etc.) and issues related to the improvement of informational mechanisms to public managers (development of practical guides, labeling schemes and environmental certification, etc.) are the main challenges to the proper execution of that public policy.

Keywords: sustainable public procurement, policy Delphi, public policy, content analysis.

\section{Introdução}

A atuação governamental, geralmente, é percebida por suas atividades-fim, os serviços públicos. E, raramente, observam-se os governos como consumidores de bens e serviços (OECD, 2000), desprezando-se sua influência sobre o mercado.

No Brasil, por exemplo, as compras governamentais movimentam recursos estimados entre 10 e 15\% do PIB (Silva e Barki, 2012). A fim de abraçar essa considerável fatia do mercado, importantes setores da economia buscam se ajustar às demandas previstas nos editais de licitação. E tais demandas podem ser coordenadas para estimular melhores práticas nos outros agentes econômicos.

E, seguindo tal raciocínio, na Cúpula Mundial sobre Desenvolvimento Sustentável de Joanesburgo, em 2002, surgiu com relevo o conceito de compras verdes que se traduz em "promover políticas de contratação pública que favoreçam o desenvolvimento e a difusão de mercadorias e serviços favoráveis ao meio ambiente" (United Nations, 2002:103). Esse foi ampliado para considerar os fatores sociais e financeiros nas tomadas de decisão de compras, chegando-se à concepção das compras públicas sustentáveis (Betiol et al., 2012).

Conforme Secchi (2010), o estudo de uma política pública, que é o caso da presente investigação, deve seguir dois preceitos fundamentais: o conhecimento legal-institucional e a 
capacidade de prever e antecipar seus efeitos sobre comportamentos individuais e coletivos. Sobre o primeiro, já existem interessantes estudos dedicados à legislação definidora da política, tais como os realizados por Santos e Barki (2011) e Oliveira e Santos (2015).

Contudo, em relação ao segundo preceito, ainda se verifica carência de investigações. Isso se justifica pois a política foi recentemente instituída, havendo poucos dados concretos para sua análise. Assim, são imprescindíveis estudos de natureza exploratória, fundados nas opiniões de especialistas sobre o assunto, capazes de aumentar o conhecimento, confirmar ou corrigir informações básicas sobre a política e estabelecer suas prioridades (BIJL, 1992).

Nesse sentido, esta pesquisa visa investigar como os especialistas compreendem, validam e refutam os objetivos já estabelecidos para a política pública e quais são as barreiras que impedem seu avanço, numa avaliação in itinere (durante a implementação) da política (Secchi, 2010), trazendo informações para o redesenho da política e futuras tomadas de decisão de seus formuladores.

\section{Revisão bibliográfica}

Embora já existam muitas experiências práticas, em diversos países, trabalhos acadêmicos nesse campo não têm sido numerosos e, portanto, as compras públicas sustentáveis ainda se constituem como uma área relativamente nova de pesquisa (Nissinen et al., 2009).

No Brasil, a situação é semelhante, não havendo estudos dedicados a avaliar de forma global a política instituída em nível federal. Os poucos trabalhos disponíveis investigam, quase sempre, as compras realizadas em instituições federais de ensino e de pesquisa (Galli, 2014; Hegenberg, 2013; Rossato, 2011; Santos, 2011).

Logo, essa política configura um tema com poucas evidências históricas e com considerável complexidade, pois envolve questões ambientais, sendo recomendável a adoção da técnica Delphi de políticas, assim como exposto por Franklin e Hart (2007). Essa técnica é utilizada, geralmente, para obter opiniões sobre um problema específico (Linstone e Turoff, 2002), sendo um instrumento facilitador na elaboração de políticas públicas e no auxílio à tomada de decisão (Hilbert et al., 2009).

A técnica busca, primordialmente, expor os diferentes posicionamentos e seus principais argumentos (prós e contras) (Stewart, 2001), mediante consulta a um grupo de especialistas, por meio de questionários que são repassados reiteradas vezes, garantindo-se o anonimato dos respondentes, a representação estatística dos resultados e o feedback das respostas do grupo (Wright e Giovinazzo, 2000). Preble (1983) assevera que o anonimato é particularmente desejável em discussões afetas ao setor público, pois as tomadas de decisão requerem opiniões de indivíduos com diversas formações e interesses. Cabe mencionar, ainda, o trabalho de Picavet e colaboradores (2012), que apresenta abordagem interessante para estabelecer níveis de consenso entre as opiniões dos especialistas.

Franklin e Hart (2007) apontam as principais vantagens da técnica: a) tempo adequado para a reflexão devido à natureza sequencial dos questionários; b) os participantes mantêm-se 
focados no problema pela comunicação estruturada; e c) reduz a influência de personalidades dominantes e os comportamentos corporativos, pois não existem debates "face a face". Pelo contrário, Wright e Giovinazzo (2000) expõem como principal desvantagem da metodologia a excessiva dependência da escolha dos respondentes nos resultados.

A técnica vem sendo utilizada na investigação de políticas públicas ambientais, assim como na priorização de problemas ambientais urbanos a serem resolvidos (Gokhale, 2001), no planejamento de ações ambientais em uma universidade pública (Wright e Sharon, 2006) ou na identificação de temas de interesse no estudo de cadeias de suprimentos sustentáveis (Seuring e Müller, 2008). Entretanto, não foram identificados registros sobre a utilização dessa técnica na avaliação das compras públicas sustentáveis.

\section{Metodologia}

\subsection{Seleção dos especialistas}

Foram selecionados 35 especialistas, por meio de amostragem seletiva, considerando-se as publicações recentes de textos científicos e os participantes de eventos relacionados com a temática ou mediante indicação dos próprios participantes (o que permitiu ampliar o rol de especialistas, inicialmente com 20 especialistas). Para capturar diferentes perspectivas, foram consultados especialistas de diversos segmentos (academia, técnicos, advocacia pública, gestão, controle e consultoria) ligados à política, como recomendado por Linstone e Turoff (2002).

\subsection{Construção dos questionários e coleta de dados}

O questionário da primeira rodada, estruturado e com questões fechadas e abertas, contemplou três seções sobre a política, nomeadamente: (1) objetivos; (2) barreiras à implementação; e (3) estratégias de incentivo. A presente publicação engloba os resultados obtidos para as duas primeiras seções. Previamente às questões, apresenta-se uma explanação sobre os objetivos da pesquisa, o tema e a metodologia empregada.

De estudos anteriores de referência sobre a política de compras sustentáveis (Santos, 2011; OECD, 2000; Hegenberg, 2013; Biderman et al., 2008; Preuss, 2009), foram extraídos nove objetivos e 15 barreiras comumente considerados em sua análise. Eles foram exibidos aos especialistas, sendo assumidos, inicialmente, como importantes na descrição da política pública. Aos especialistas coube avaliar se tal assunção inicial coincide com suas opiniões, utilizando-se uma escala de Likert (níveis: 1. irrelevante; 2. pouco importante; 3. importante; 4. muito importante). Não foram admitidas respostas neutras, mas permitiu-se a não apresentação de resposta. 
O convite aos especialistas ocorreu por meio de correio eletrônico, com o envio de até quatro lembretes (caso não houvesse resposta, em intervalos de 20 dias), no período de agosto a setembro de 2013. A taxa de resposta foi de 48,6\% (17 respostas).

O questionário para a segunda rodada, enviado de fevereiro a maio de 2014, diferenciouse do anterior para mostrar a cada respondente: (a) sua resposta na primeira rodada; (b) a mediana e o intervalo interquartil (entre o $1^{\circ}$ e o $3^{\circ}$ quartis) das respostas dadas por todos os especialistas para cada item; e (c) campo para que o respondente cuja resposta ficou fora do intervalo interquartil apresentasse os argumentos que motivaram sua resposta, com o intento de obter os argumentos extremos dos especialistas, capturando os prós e os contras relativos a cada item.

Adicionalmente, permitiu-se que os especialistas modificassem sua resposta inicial com base nas respostas dos outros e sugerissem a inclusão de novos itens. A taxa de resposta, nessa rodada, alcançou 70,6\% (12 respostas, trazendo 72 comentários relativos às opiniões dos especialistas quanto aos itens dispostos no questionário). Os comentários dos especialistas são identificados por meio dos segmentos a que pertencem (academia - AC; técnicos -TC; advocacia pública - AD; gestão, controle e consultoria - CG). A metodologia aqui adotada assemelhase àquela utilizada por Wright e Sharon (2006).

\section{Resultados e discussões}

A análise dos resultados seguiu dois caminhos: a) a análise quantitativa das respostas, nos termos de Picavet e colaboradores (2012), classificando-se os itens de cada seção conforme o nível de consenso e a mediana, nessa ordem, apresentando-se, também, os intervalos interquartis; e b) a análise qualitativa das opiniões trazidas pelos especialistas, utilizando-se a análise de conteúdo (Bardin, 2009).

O nível de consenso representa o percentual de respostas que concordam com as afirmações iniciais (ou seja, que dado objetivo ou barreira é considerado "importante" ou "muito importante" para o especialista). Os níveis de consenso foram predefinidos: menor que 65\% (dissenso), 75\% (consenso modesto), 80\% (consenso) e 85\% (forte consenso). Serão apresentados os resultados consolidados após a segunda rodada, momento em que foi atingido significativo consenso, dispensando-se a realização de mais rodadas.

Os comentários, por sua vez, foram analisados em três etapas: 1) pré-análise, que consiste na retomada das hipóteses e dos objetivos iniciais do estudo e na familiarização com os dados brutos, por meio da leitura e da releitura dos comentários, com a formação de categorias de análise; 2) exploração do material, com a subdivisão dos comentários nas categorias de análise já constituídas; e 3) tratamento dos dados, inferência e interpretação, com a interpretação de cada categoria e a retirada de trechos relevantes dos comentários.

\subsection{Objetivos da política pública}

Na tabela 1, encontram-se os níveis de consenso e as medianas para cada um dos objetivos da política pública. 
Tabela 1

Classificação dos objetivos da política pública

\begin{tabular}{|lcc|}
\hline Objetivos da política pública & Nível de consenso & Respostas* $^{*}$ \\
\hline Garantir um meio ambiente saudável para as gerações futuras & $100 \%$ & $4(4-4)$ \\
Promover práticas de produção mais sustentáveis e reconhecer aquelas já existentes & $100 \%$ & $4(4-4)$ \\
Alcançar maior performance ambiental, à medida que a relação produto/resíduo diminui & $100 \%$ & $3(3-4)$ \\
Adequar-se à legislação e a outros requisitos normativos & $94,1 \%$ & $4(3-4)$ \\
Promover práticas de consumo sustentável na população & $94,1 \%$ & $4(3-4)$ \\
Induzir a adoção de padrões de consumo sustentável em organizações privadas & $90,9 \%$ & $4(3-4)$ \\
Promover maior efetividade a outras políticas ambientais sem a realização de novos gastos & $90 \%$ & $3,5(3-4)$ \\
Conscientizar os servidores nas questões ligadas à sustentabilidade & $88,2 \%$ & $4(3-4)$ \\
Incentivar a inovação tecnológica & $88,2 \%$ & $3(3-4)$ \\
Melhorar a imagem institucional & $88,2 \%$ & $3(3-3)$ \\
Obter ganhos de escala & $88,2 \%$ & $3(3-3)$ \\
Respeitar direitos humanos e sociais & $75 \%$ & $3,5(2,75-4)$ \\
Incentivo às micro e pequenas empresas & $72,7 \%$ & $3(2,5-4)$ \\
Fomento à transparência na administração pública & $70 \%$ & $4(2,25-4)$ \\
Inclusão de catadores de materiais recicláveis & $30 \%$ & $2(2-2,75)$ \\
\hline
\end{tabular}

Fonte: Dados da pesquisa.

* As respostas na forma $M(X-Y)$ correspondem à mediana $(M)$ das opiniões dos especialistas e ao intervalo interquartil $(X-Y)$, ou seja, a dispersão de tais opiniões.

Analisando-se, conjuntamente, os resultados quantitativos trazidos na tabela 1 e os argumentos expostos pelos especialistas, foram derivadas duas categorias de análise relevantes para a definição dos objetivos da política: 1) Dimensões da sustentabilidade; e 2) Integração de políticas públicas.

\subsubsection{Dimensões da sustentabilidade}

Há consenso de que a sustentabilidade pode ser decomposta em três dimensões essenciais: ambiental, social e econômica, e, portanto, qualquer política que atue em prol dela deve equilibrar tais dimensões. Todavia, em relação às compras públicas sustentáveis, identifica-se que há, comumente, a prevalência da dimensão ambiental sobre as outras duas (Gomes, 2013).

Tal tendência também foi notada no presente estudo: observa-se que aqueles objetivos ligados à dimensão ambiental ("Garantir um meio ambiente saudável para as gerações futuras") obtiveram níveis de consenso e medianas superiores àqueles objetivos ligados às outras dimensões, tais como: "Obter ganhos de escala" (dimensão econômica) e "Respeitar direitos individuais e sociais" (dimensão social). A partir dos comentários de alguns especialistas, con- 
firma-se esse entendimento:

Não me parece ser este um objetivo da Política de Compras Públicas Sustentáveis [...] o respeito aos direitos individuais e sociais. [TC2]

[...] o poder de compra governamental não deve ser entendido como um objetivo, mas um meio de incentivar melhores práticas ambientais. [TC3]

\subsubsection{Integração de políticas públicas}

Segundo Baumol (1988), uma dada política é eficiente quando está integrada a um conjunto de outras políticas que, em regra, potencializarão suas vantagens e evitarão suas fraquezas individuais. A política pública de compras sustentáveis é tratada assim pelo governo federal, em seu Plano de Ação para a Produção e o Consumo Sustentáveis (Brasil, 2010), integrada às políticas de resíduos sólidos, de mudanças climáticas e de desenvolvimento produtivo.

Da tabela 1, observa-se que, em relação às políticas ambientais, há tal consciência pelos especialistas da necessidade da integração das políticas ( $90 \%$ de nível de consenso). No entanto, os especialistas não visualizam a necessidade de integração quando ela deveria ocorrer com políticas não essencialmente ambientais:

Apoio a pequenas e microempresas é requisito importante, mas não deve ser o foco de uma política que envolva sustentabilidade. [CG2]

Não parece-me adequado incluir catadores numa política de compras públicas sustentáveis, pois este público já é tratado em política relacionada a resíduos. [TC2]

\subsection{Barreiras à implementação da política pública}

Da tabela 2, obtêm-se os níveis de consenso e a mediana para cada barreira tida, inicialmente, como importante obstáculo à implementação da política pública.

Tabela 2

Classificação das barreiras à implementação da política pública

\begin{tabular}{|lcc|}
\hline Barreiras à implementação & $\begin{array}{c}\text { Nível de } \\
\text { consenso }\end{array}$ & Respostas* \\
\hline $\begin{array}{l}\text { Falta de capacitação dos servidores envolvidos } \\
\text { A cultura organizacional das instituições públicas, ainda resistente a questões ligadas à } \\
\text { sustentabilidade }\end{array}$ & $100 \%$ & $4(4-4)$ \\
$\begin{array}{l}\text { Elevada complexidade das decisões a serem tomadas pelos gestores de compras } \\
\text { Ausência de catálogos de materiais e/ou serviços com critérios específicos para a escolha } \\
\text { de itens sustentáveis }\end{array}$ & $88,2 \%$ & $4(3-4)$ \\
Ausência de informações confiáveis sobre os impactos ambientais de produtos e serviços & $82,4 \%$ & $3(3-4)$ \\
\hline
\end{tabular}




\begin{tabular}{|lcc|}
\hline Barreiras à implementação & $\begin{array}{c}\text { Nível de } \\
\text { consenso }\end{array}$ & Respostas* \\
\hline $\begin{array}{l}\text { Incerteza acerca das possibilidades legais de se incluir critérios ambientais nos editais de } \\
\text { licitação }\end{array}$ & $82,4 \%$ & $4(3-4)$ \\
Ausência de esquemas de avaliação da conformidade (certificação, rotulagem etc.) & $81,3 \%$ & $3(3-4)$ \\
Escassez de produtos/serviços sustentáveis no mercado & $81,3 \%$ & $3(3-4)$ \\
$\begin{array}{l}\text { Falta de incentivo governamental (financiamentos) à inovação tecnológica, para produtos } \\
\text { "verdes" }\end{array}$ & $80 \%$ & $3(3-4)$ \\
$\begin{array}{l}\text { Não haver previsão legal para a utilização do critério sustentável como critério de } \\
\text { desempate ou como margem de preferência }\end{array}$ & $70,6 \%$ & $3(2-3)$ \\
Os preços de mercado usualmente superiores das contratações sustentáveis & $68,8 \%$ & $3(2-4)$ \\
Resistência dos órgãos de controle (TCU, CGU etc.) à adoção de critérios ambientais & $64,7 \%$ & $3(2-4)$ \\
Impedimento legal para a utilização de certificações ISO de gestão ambiental como & $58,8 \%$ & $3(2-3)$ \\
critérios de habilitação & $58,8 \%$ \\
Normatização insuficiente do assunto & $3(2-4)$ \\
O conflito entre a inclusão de critérios de sustentabilidade e a restrição da competitividade & $58,8 \%$ & $3(2-3)$ \\
Falta de acesso às normas que definem qualidade ambiental & $56,3 \%$ & $3(2-3)$ \\
\hline
\end{tabular}

Fonte: Dados da pesquisa.

Tais resultados, em conjunto com os argumentos trazidos pelos especialistas, conduzem à formação de três categorias de análise: (1) Aspectos institucionais; (2) Conhecimento e informação; e (3) Aspectos econômicos, às quais se assemelham às obtidas por Rossato (2011).

\subsubsection{Aspectos institucionais}

Essa categoria engloba as questões relativas à mudança de cultura organizacional, por meio da conscientização e da capacitação de servidores. Os especialistas são unânimes ao afirmar que a resistência às questões ligadas à sustentabilidade ainda se constitui no principal obstáculo à efetividade da política de compras sustentáveis:

[...] a capacitação é importante, mas não é algo tão difícil ou complicado, é mais difícil mudar a cultura organizacional para absorver as CPS como parte da rotina de compras públicas na instituição. [AC1]

\subsubsection{Conhecimento e informação}

Tal categoria abrange as formas de conhecimento e informação necessárias para a adequada realização de compras sustentáveis. Aqui, foram observadas opiniões divergentes entre os especialistas. Alguns consideram que ainda são necessários avanços nesse quesito, reportando carências de informações confiáveis: 
Reforço a importância fundamental de manuais públicos gratuitos para os gestores e capacitações sobre o tema. [AD2]

[...] Não vejo qualquer obstáculo a viabilizar a criação de um banco de produtos sustentáveis. [CG3]

[...] devem existir treinamentos sobre o significado dessas certificações e sobre quais podem ser usadas nas licitações. [AC2]

Outros, por sua vez, avaliam que as informações já existem, mas faltam interesse ou capacidade para bem utilizá-las:

Sobra informação e falta vontade... Basta clicar no Google que um mundo de informação vem de graça! [TC4]

Entendo que não é difícil o acesso às normas, desde que se saiba qual utilizar. [CG4]

Adicionalmente, foi travado outro diálogo no âmbito dessa categoria. Alguns especialistas afirmaram que a complexidade da legislação brasileira sobre o tema e a resistência dos órgãos de controles (CGU e TCU) trazem insegurança para o gestor público em suas decisões de compra. Mas, de forma majoritária, os especialistas refutaram essa compreensão. Duas opiniões, em especial, são bastante exemplificativas:

Este argumento é bastante usado pelos compradores públicos, mas hoje em dia muito mais por resistência a uma mudança em suas práticas de compras do que em razão de uma barreira tão relevante. [CG1]

[...] ainda subsiste um "mito" no setor público no sentido de que o TCU é contrário às licitações sustentáveis, oriundo de falta de conhecimento. [AD2]

\subsubsection{Aspectos econômicos}

Nessa última categoria estão as discussões relativas às barreiras de cunho econômico, com destaque para os efeitos da inclusão de critérios de sustentabilidade sobre a manutenção da competitividade. Se um gestor de compras decide, por exemplo, exigir numa licitação que determinado padrão ambiental deva ser cumprido, inevitavelmente haverá um menor número de fornecedores capazes de atender ao que a administração deseja e, consequentemente, uma elevação dos preços de mercado. Alguns especialistas (posição minoritária) se identificaram com essa compreensão:

Este aspecto pareceu-me relevante, pois dependendo dos critérios adotados, pode haver direcionamento para apenas algumas empresas do mercado e a redução da competitividade. [TC2]

A contrario sensu, a maioria dos especialistas se posicionou pela não incompatibilidade entre compras sustentáveis e a manutenção da competitividade, desde que exista equilíbrio nas exigências formuladas, de tal modo que o papel indutor do Estado para o aparecimento de 
novas tecnologias não se transforme em direcionamento das licitações para poucos fornecedores. Sobre a elevação de preços, há apontamentos no sentido de que a administração deve considerar os preços implícitos associados à qualidade ambiental dos produtos adquiridos. Algumas opiniões merecem destaque:

A licitação sustentável não restringe a competição [...]. O que há é desconhecimento de "como fazer" para não restringir a competição. [AD2]

Os preços de mercado devem ser avaliados em conjunto com os custos de manutenção e reposição, além dos custos ambientais e sociais oriundos de externalidades negativas provocadas por produtos e processos produtivos menos eficientes. [TC3]

\section{Conclusões}

Aqui, foram analisadas as diferentes opiniões dos especialistas na política de compras públicas sustentáveis, de modo a ampliar o conhecimento sobre elas, identificando os objetivos e os desafios considerados prioritários, por meio de um Delphi de políticas. Dessa forma, foi verificado se as diretrizes expostas na legislação definidora da política são validadas ou refutadas pelos especialistas, dando vazão para a necessidade de novas investigações e à proposição de mecanismos para a superação de obstáculos à sua efetivação.

Dos resultados obtidos, conclui-se que, ao contrário da legislação que é definida para a política, os especialistas compreendem as compras públicas sustentáveis como uma política ambiental stricto sensu, ou seja, que considera apenas aspectos de natureza ambiental em sua formulação, coincidindo com estudo anterior desenvolvido em Portugal (Gomes, 2013). Do ponto de vista prático, cabe aos formuladores da política estabelecer estratégias de comunicação que reforcem seus aspectos sociais, tais como: a inclusão de catadores, por meio da coleta seletiva solidária ou o estabelecimento de mecanismos que assegurem o pagamento de verbas trabalhistas a funcionários terceirizados, nos casos em que a empresa contratada deixe de fazê-lo.

Similarmente, verificou-se que os especialistas não identificam como necessária a integração das compras públicas sustentáveis a outras políticas públicas de cunho não ambiental, o que vai de encontro ao prescrito na legislação, onde é tida como um dos eixos das políticas voltadas para a sustentabilidade na administração pública, com atuação integrada. Nesse ponto, vê-se que a administração pública federal já vem estabelecendo estratégias para disseminar essa cultura de integração das políticas públicas. Inclusive, houve recente edição do Decreto $\mathrm{n}^{\circ} \mathbf{0}$ 7.746/2012 com o objetivo de integrar o conjunto de políticas públicas ligadas à sustentabilidade no setor público, com base no conceito de logística sustentável.

Na sequência, foram categorizadas as barreiras à implementação, donde foram derivadas três categorias de análise, quais sejam: aspectos institucionais; conhecimento e informa- 
ção; e aspectos econômicos. Essas categorias indicam aos formuladores da política quais são os caminhos prioritários a serem percorridos na busca por uma maior efetividade da política pública. Por sua vez, à academia, indicam quais são os temas que devem ser preponderantemente avaliados em investigações científicas futuras.

Uma das categorias chama a atenção: aquela denominada "Conhecimento e informação", que reclama a necessidade de análises e ações ligadas à criação de instrumentos capazes de trazer informação aos gestores de compras (que, na prática, implementam a política). Cabe frisar a posição majoritária dos especialistas ao afirmar que já existem informações suficientes, mas que há falta de interesse e capacidade para sua correta utilização.

\section{Referências}

BARDIN, Laurence. Análise de conteúdo. Lisboa: Edições 70, 2009.

BAUMOL, William J. The theory of environmental policy. Cambridge University Press, 1988.

BETIOL, Luciana S. et al. Compra sustentável: a força do consumo público e empresarial para uma economia verde e inclusiva. São Paulo: Editora FGV, 2012.

BIDERMAN, Rachel et al. Guia de compras públicas sustentáveis: uso do poder de compra do governo para a promoção de desenvolvimento sustentável. 2. ed. Rio de Janeiro: FGV, 2008.

BIJL, Rob. Delphi in a future scenario study on mental health and mental health care. Futures, v. 24, n. 3, p. 232-250, 1992.

BRASIL, Ministério do Meio Ambiente. Plano de ação para a produção e o consumo sustentáveis: versão para consulta pública. 2010.

FRANKLIN, Kathy K.; HART, Jan K. Ideas generation and exploration: benefits and limitations of the policy Delphi research method. Innovative Higher Education, v. 31, n. 4, p. 237-246, 2007.

GALLI, Alessandra. Compras públicas sustentáveis na UTFPR: estudo de caso do campus Curitibasede reitoria. Tese (doutorado em tecnologia) - Universidade Tecnológica Federal do Paraná, Curitiba, 2014.

GOMES, Manuel S. Sustainable public procurement in Portugal-State of the art and future prospects. Eur. Pub. Private Partnership L. Rev., v. 8, n. 1, p. 60-72, 2013.

GOKHALE, Anu A. Environmental initiative prioritization with a Delphi approach: a case study. Environmental management, v. 28, n. 2, p. 187-193, 2001.

HEGENBERG, Juliana T. As compras públicas sustentáveis no Brasil: um estudo nas universidades federais. Dissertação (mestrado em planejamento e governança pública) — Universidade Tecnológica Federal do Paraná, Curitiba, 2013. 
HILBERT, Martin et al. Foresight tools for participative policy-making in inter-governmental processes in developing countries: Lessons learned from the eLAC Policy Priorities Delphi. Technological Forecasting and Social Change, v. 76, n. 7, p. 880-896, 2009.

LINSTONE, Harold A.; TUROFF, Murray. The Delphi method: techniques and applications. Nova York: Addison Wesley, 2002.

NISSINEN, Ari et al. Environmental criteria in the public purchases above the EU threshold values by three Nordic countries: 2003 and 2005. Ecological Economics, v. 68, n. 6, p. 1838-1849, 2009.

OLIVEIRA, Bernardo Carlos S. C. M.; SANTOS, Luis Miguel L. Compras públicas como política para o desenvolvimento sustentável. Rev. Adm. Pública, Rio de Janeiro, v. 49, n. 1, p. 189-206, 2015.

ORGANIZATION FOR ECONOMIC CO-OPERATION AND DEVELOPMENT. OECD. Greener public purchasing: issues and practical solutions. Paris: OECD Publications, 2000.

PICAVET, Eline et al. Evaluating and improving orphan drug regulations in Europe: a Delphi policy study. Health Policy, v. 108, n. 1, p. 1-9, 2012.

PREBLE, John F. Public sector use of the Delphi technique. Technological Forecasting and Social Change, v. 23, n. 1, p. 75-88, 1983.

PREUSS, Lutz. Addressing sustainable development through public procurement: the case of local government. Supply Chain Management: An International Journal, v. 14, n. 3, p. 213-223, 2009.

ROSSATO, Jaqueline. Compras públicas sustentáveis: estudo nas instituições federais de ensino superior a partir das comissões permanentes de licitação. Dissertação (mestrado em administração) — Universidade Federal de Santa Catarina, Florianópolis, 2011.

SANTOS, Rejane M. T. Compras públicas sustentáveis: a utilização do poder de compra do Estado no fomento de produtos ecologicamente corretos na Fiocruz. Dissertação (mestrado profissional em saúde pública) — Escola Nacional de Saúde Pública, Rio de Janeiro, 2011.

SANTOS, Murillo G.; BARKI, Teresa V. P. (Coord.). Licitações e contratações públicas sustentáveis. Belo Horizonte: Fórum, 2011.

SECCHI, L. Políticas públicas: conceitos, esquemas de análise, casos práticos. São Paulo: Cengage Learning, 2010.

SEURING, Stefan; MÜLLER, Martin. Core issues in sustainable supply chain management — a Delphi study. Business Strategy and the Environment, v. 17, p. 455-466, 2008.

SILVA, Renato C.; BARKI, Teresa V. P. Compras públicas compartilhadas: a prática das licitações sustentáveis. Revista do Serviço Público, v. 63, n. 2, p. 157-175, 2012.

STEWART, J. Is the Delphi technique a qualitative method? Medical Education, v. 35, n. 10, p. 922-923, 2001.

UNITED NATIONS. Report of the world summit on sustainable development - Johannesburg, South Africa. 26 ago./4 set. 2002. 
WRIGHT, James T. C.; GIOVINAZZO, Renata A. Delphi: uma ferramenta de apoio ao planejamento prospectivo. Caderno de Pesquisas em Administração, v. 1, n. 12, p. 54-65, 2000.

WRIGHT, Tarah; SHARON, Alexandra. Giving "teeth" to an environmental policy: a Delphi study at Dalhousie University. Journal of Cleaner Production, v. 14, n. 9, p. 761-768, 2006.

Hugo Leonnardo Gomides do Couto é analista de planejamento, gestão e infraestrutura da Fundação Instituto Brasileiro de Geografia e Estatística (IBGE). Doutor em ciências ambientais pela Universidade Federal de Goiás (UFG). E-mail: hlcouto@gmail.com.

Francis Lee Ribeiro é professora adjunta da Universidade Federal de Goiás (UFG). Doutora em economia aplicada pela Universidade de Viçosa (UFV). E-mail: francisleerib@gmail.com. 